\title{
Tratamiento cognitivo-conductual en trastornos de ansiedad infantil
}

\section{Cognitive-behavioral treatment of children anxiety disorders}

\author{
Alejandra Viridiana Gutiérrez García ${ }^{1}$, Mariana Gutiérrez Lara², \\ Cecilia Méndez Sánchez ${ }^{3}$ y Angélica Riveros Rosas ${ }^{4}$
}

\begin{abstract}
RESUMEN
El propósito de esta investigación fue evaluar el efecto del tratamiento cognitivo-conductual en un grupo de niños con trastornos de ansiedad, de entre 8 y 11 años de edad, pacientes de un hospital psiquiátrico de la Ciudad de México. Los casos se atendieron de manera individual bajo un diseño compuesto por tres fases: evaluación, tratamiento cognitivo-conductual y seguimiento. Se aplicaron asimismo un pretest al inicio del procedimiento, un postest al término y dos seguimientos al mes y a los dos meses de concluido aquel, mediante los instrumentos CMASR-2 y SCAS, validados y estandarizados en México. Los datos se compararon entre sí y se representaron de forma grupal a través de diagramas de caja y bigote, además de que se analizaron bajo la prueba de rangos con signo de Wilcoxon. Los resultados indican que el tratamiento cognitivo-conductual generó una disminución de las creencias distorsionadas que los pacientes tenían respecto a los estímulos disparadores de ansiedad, así como de la sintomatología fisiológica asociada al padecimiento. Se disminuyeron las puntuaciones naturales obtenidas a lo largo de las mediciones realizadas y el cambio resultó estadísticamente significativo.
\end{abstract}

Palabras clave: Ansiedad; Niños; Tratamiento cognitivo-conductual, Pretest-postest; Seguimiento.

\begin{abstract}
This study's purpose was to evaluate the effectiveness of Cognitive Behavioral Treatment (CBT) in children with anxiety disorders aged eight to eleven years, who were patients of a psychiatric hospital in Mexico City. Cases were intervened individually through a treatment scheme composed by three phases: Evaluation, Cognitive Behavioral Treatment, and Follow-up. Pretest measures were collected before therapy, as well as at the end, and follow up measurements at one and two months after finishing treatment. Measures included the CMASR-2 and SCAS instruments, validated and standardized in Mexico. Data, represented in a boxplot were analyzed with the Wilcoxon statistical test for group results. Results show that CBT led to a reduction of distorted beliefs and patients' anxiety-triggering stimuli. Disorder associated physiological anxiety symptoms were also reduced. Cognitive Behavioral Treatment generated a reduction of scores throughout the process, and changes were statistically significant.
\end{abstract}

Key words: Anxiety; Children; Cognitive-behavioral treatment; Pretest-posttest; Follow up.

\footnotetext{
${ }^{1}$ Universidad Nacional Autónoma de México y Hospital Psiquiátrico Infantil “Dr. Juan N. Navarro", Unidad Adolescentes Mujeres, Av. San Buenaventura 86, Col. Belisario Domínguez Secc. 16, Del. Tlalpan, 14080 Ciudad de México, México, tel. (55)55-73-48-66, ext. 155, correo electrónico: alejandragtzg@hotmail.com. Artículo recibido el 7 de agosto y aceptado el 10 de noviembre de 2017.

${ }^{2}$ Facultad de Psicología, Edif. C, Cubículo 36, Universidad Nacional Autónoma de México, Av. Universidad 3004, Col. Copilco-Universidad, Del. Coyoacán, 04510 Ciudad de México, México, tel. (55)56-22-23-15, correo electrónico: marianagutierrezlara@gmail.com.

${ }^{3}$ Departamento de Psicología, Edif. 212, Cubículo 1 (planta baja), Universidad Autónoma de Aguascalientes. Av. Universidad 940, Ciudad Universitaria, 20131 Aguascalientes, Ags., México, tel. 910-84-91 y 910-74-87, correo electrónico: ceciliams2002@yahoo.com.mx.

${ }^{4}$ Facultad de Contaduría y Administración, División de Investigación, Cubículo 31, Universidad Nacional Autónoma de México, Circuito exterior s/n, Ciudad Universitaria, Del. Coyoacán, 04510 Ciudad de México, México, tel. (55)56-22-54-87, ext. 131, correo electrónico: ariveros@, fca.unam.mx.

Citación: Gutiérrez G., A.V., Gutiérrez L., M., Méndez S., C. y Riveros R., A. (2018). Tratamiento cognitivo-conductual en trastornos de ansiedad infantil. Psicología y Salud, 28(2), 177-186.
} 
$\mathrm{D}$ e acuerdo con los cambios que la sociedad ha experimentado en los últimos años, se han observado distintas afectaciones conductuales y afectivas en las personas. De manera específica, la población infantil ha mostrado síntomas de considerable importancia, tales como preocupaciones constantes, dificultad para quedarse sola, pesadillas y enfermedades médicas (gastritis y colitis nerviosas), entre otras. Según la literatura (American Psychiatric Association [APA], 2013; Beesdo, Knappe y Pine, 2009), tales características corresponden al concepto de "ansiedad", que es una emoción que se genera bajo un estímulo que el individuo interpreta como peligroso y que se relaciona con preocupaciones, sentimientos de tensión y sensaciones corporales, como el aumento de la presión arterial, por lo que el individuo buscará escapar activamente y protegerse de la amenaza.

Cuando estos síntomas aparecen en el individuo de manera frecuente, severa y persistente, se pueden cumplir criterios para diagnosticar un trastorno de ansiedad (Benjamin et al., 2010). De acuerdo con Polanczyk, Salum, Sugaya, Caye y Rohde (2015), la prevalencia de este trastorno en la población infantil y adolescente es alarmante, pues de $13.4 \%$ de niños y adolescentes con trastornos mentales en el mundo, $6.5 \%$ se cataloga con ese trastorno, cuya incidencia constituye el primer lugar en esta población.

En México, de acuerdo con las estadísticas del Hospital Psiquiátrico Infantil "Dr. Juan N. Navarro" (2014), 103 niños de entre 5 y 14 años de edad fueron atendidos durante el año 2014 por diagnóstico de trastorno de ansiedad generalizada, constituyéndose así en la cuarta causa de consulta externa después de los trastornos no especificados, las perturbaciones de la actividad y la atención, y los episodios depresivos moderados.

Este tipo de psicopatología también es relevante en la población adulta, pues según la Encuesta Nacional de Epidemiología Psiquiátrica (Medina-Mora et al., 2003), 28.6\% de la población de entre 18 y 65 años de edad ha manifestado algún trastorno mental a lo largo de su vida, de los cuales $14.3 \%$ corresponde a los trastornos de ansiedad, siendo estos los de mayor frecuencia. Es sabido también que dichos trastornos tienen un inicio temprano durante los dos periodos de vida previos (Keeton, Kolos y Walkup, 2009), por lo que la infancia y la adolescencia se convierten en un importante foco de atención para la prevención y el tratamiento oportunos y así impedir su desarrollo en etapas posteriores de la vida (World Health Organization [WHO], 2004).

Respecto al tratamiento, de acuerdo con la Medicina Basada en Evidencias (MBE), se ha demostrado la efectividad de la terapia cognitivo-conductual (TCC en lo sucesivo) como primera opción para mejorar la sintomatología, pero cuando esta se combina con farmacoterapia - principalmente sertralina-, se reducen aún más los síntomas (Creswell, Waite y Cooper, 2014; Sawyer y Nunez, 2014). Hay en México algunos estudios que han concluido que la TCC mejora también la severidad del trastorno, entre los que se encuentran las intervenciones en niños con ansiedad social (Gil y Hernández, 2009), los tratamientos grupales e individuales y los estudios de caso (Gutiérrez y Gutiérrez, 2015; López, 2012; Peña y Ramos, 2009); sin embargo, se reconoce la escasa generalización de los hallazgos y la falta de niveles altos de confiabilidad, de acuerdo con la MBE.

En cuanto a la farmacoterapia, los inhibidores selectivos de la recapturación de serotonina (ISRS), tales como la sertralina, la fluoxetina y la fluvoxamina, son efectivos para tratar esta psicopatología (Ipser, Stein, Hawkridge y Hoppe, 2009); pese a ello, la preocupación radica en el tiempo necesario de ingesta (un año a partir de que se observa una mejoría de 50\%) y los efectos secundarios, tales como la activación de síntomas de trastorno por déficit de atención con hiperactividad (TDAH), ataxia e irritabilidad, por solo mencionar algunos (Cárdenas, Feria, Palacios y De la Peña, 2010; Creswell et al., 2014; Martínez, Fernández, Navarro y Martínez, s. f.; Reid et al., 2015).

Derivado de este hecho, Creswell y Waite (2016) subrayan la necesidad de llevar a cabo análisis de costo-beneficio respecto al tratamiento, y sugieren además que la primera línea de elección sea una intervención de bajo nivel invasivo mediante la TCC; en el caso de que los síntomas persistan, la segunda línea es el mismo tipo de tratamiento, pero de mayor duración y con monitoreo constante; la tercera, en caso de solo notarse una mejoría parcial, consiste en insertar sesiones de mantenimiento o refuerzo, y la cuarta y última es 
la aplicación de farmacoterapia al haberse agotado ya las posibilidades anteriores.

En el caso específico del tratamiento, se ha reconocido que en las instituciones de salud existe una carencia de protocolos de evaluación e intervención de los trastornos mentales que puedan funcionar como guía de los psicólogos clínicos (Sawyer y Nunez, 2014). En México, solo 30\% cuenta con ellos (Secretaría de Salud, Organización Panamericana de la Salud y Organización Mundial de la Salud, 2011). Lo anterior cobra especial importancia ya que, al no haber tales protocolos, los profesionales de la psicología invierten una mayor cantidad de tiempo y esfuerzo en la planeación de tratamientos efectivos para este tipo de padecimientos, cuya replicabilidad por parte de otros profesionales resulta particularmente compleja.

Con base en lo anterior, este trabajo buscó evaluar el efecto que tiene el TCC en niños escolares con trastornos de ansiedad para, en el futuro, poder plantear guías clínicas de tratamiento que sean efectivas en la población infantil mexicana.

\section{MÉTODO}

\section{Objetivo}

El objetivo del presente estudio fue evaluar el efecto del TCC en un grupo de 10 niños de 8 a 11 años de edad con trastornos de ansiedad, pacientes de un hospital psiquiátrico de la Ciudad de México.

La pregunta de investigación planteada fue si es efectivo el TCC en niños con trastornos de ansiedad. La hipótesis que subyace es que esta forma de tratamiento, aplicado a ese tipo de población con dicho padecimiento, generará una disminución de la sintomatología ansiosa.

\section{Diseño}

Para ello, se utilizó un diseño pretest-postest con seguimientos en cada uno de los casos, con tres fases en la atención psicológica de los pacientes: la primera fue la evaluación y la medición de la ansiedad; la segunda, relativa a la intervención con TCC y la participación de los cuidadores primarios, y la tercera, correspondiente al seguimiento, en donde se hicieron tres evaluaciones: la primera al término del tratamiento (postest), seguida por la segunda y la tercera, con un mes de diferencia entre ellas. El análisis grupal de los datos se hizo con la prueba de rangos con signo de Wilcoxon. El muestreo fue de tipo no probabilístico accidental.

\section{Participantes}

Se trabajó con diez niños de 8 a 11 años de edad, de los cuales nueve fueron varones y una mujer, todos ellos pacientes de la institución ya referida. Estos casos presentaron características sintomatológicas cuyo diagnóstico correspondiente fue algún trastorno de ansiedad, establecido por su médico paidopsiquiatra tratante y confirmado por las escalas de Ansiedad Manifiesta en Niños Revisada 2 (CMASR-2) (Reynolds y Richmond, 2012) y la de Ansiedad para Niños de Spence (SCAS) (Spence, 1997; cf. Hernández et al., 2010). Todos los participantes estaban ingiriendo algún medicamento psiquiátrico para tratar su condición, como sertralina o fluoxetina. Se consideró que la farmacoterapia surtiría efecto trascurridas cuatro o seis semanas, por lo que en seis pacientes tal efecto ocurrió durante la fase de evaluación; en uno sucedió durante la fase de tratamiento, y tres pacientes no presentaron modificación alguna debido al medicamento a lo largo del proceso, pues el efecto había ocurrido aproximadamente un mes antes de que comenzara la atención psicológica. Nueve de los diez participantes tenían diagnóstico de ansiedad comórbida con TDAH. En el estudio participaron también los cuidadores primarios de los niños (la madre, ambos padres o la abuela).

\section{Instrumentos y herramientas}

Escala de Ansiedad Manifiesta en Niños Revisada 2 (CMASR-2) (Reynolds y Richmond, 2012). Este instrumento consta de 49 reactivos, cada uno de ellos con dos opciones de respuesta (Sí/No), y evalúa el nivel y naturaleza de la ansiedad en personas de entre 6 y 19 años de edad. En su estandarización mexicana obtuvo un coeficiente alfa de confiabilidad de 0.87 .

Escala de Ansiedad para niños de Spence (SCAS) (Spence, 1997; Hernández et al., 2010). Es un instrumento de autoinforme que mide ansiedad en niños de entre 8 y 12 años de edad, el cual cons- 
ta de 44 reactivos, los cuales se contestan en una escala tipo Likert de cuatro opciones de respuesta que van de "Nunca" a "Siempre". La versión para México tiene un coeficiente alfa de Cronbach de 0.88 .

A lo largo del proceso se utilizaron diferentes juegos y materiales que permitieron cumplir con el objetivo terapéutico propuesto durante las sesiones. La Tabla 1 muestra una descripción de dichos juegos y de la forma en que se emplearon con los niños a lo largo del procedimiento.

\section{Procedimiento}

En el Servicio de Psicoterapia Cognitivo-Conductual de la institución se identificó a los diez par- ticipantes que cumplían con los criterios de inclusión del estudio, quienes desde su admisión al hospital contaban con el diagnóstico del paidopsiquiatra tratante y con una farmacoterapia acorde con aquel, pero con una mejoría parcial clínicamente observada, por lo que se decidió la interconsulta a Psicología.

El procedimiento planteado se llevó a cabo de manera individual con cada uno de los niños y con la participación de sus respectivos cuidadores. Se establecieron las sesiones psicoterapéuticas a razón de una vez por semana, cada una de ellas con una duración de 60 minutos. Hubo un total de 19 sesiones en el programa total, las que se ajustaron en cada caso según el problema presentado.

Tabla 1. Herramientas empleadas durante el proceso.

\begin{tabular}{|c|c|c|}
\hline Herramienta & Descripción & Ejemplos \\
\hline Juegos & $\begin{array}{l}\text { Juegos de estrategia y desafíos cognitivos que } \\
\text { contenían tarjetas ordenadas por dificultad y que } \\
\text { el jugador debía resolver con base en ciertas re- } \\
\text { glas propuestas. }\end{array}$ & 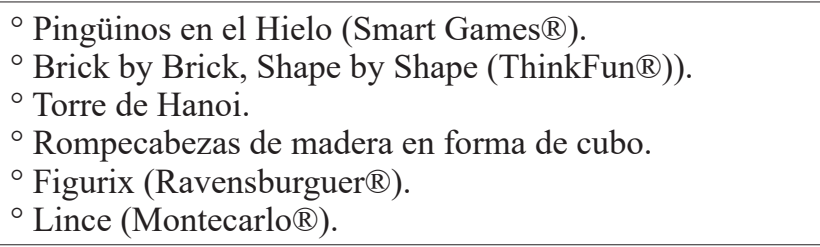 \\
\hline Otros & $\begin{array}{l}\text { Material de apoyo para complementar los objeti- } \\
\text { vos de las sesiones con el niño. }\end{array}$ & $\begin{array}{l}{ }^{\circ} \text { Disfraces (peluca, rebozo, diadema de reno, cola de dia- } \\
\text { blo, collares de colores). } \\
{ }^{\circ} \text { El pastel de la responsabilidad (rebanadas de unicel de } \\
\text { diferentes tamaños que al juntarlos forman un pastel). } \\
{ }^{\circ} \text { Papelería y hojas blancas, lápices y lápices de colores. }\end{array}$ \\
\hline
\end{tabular}

A lo largo del proceso se llevaron a cabo las siguientes fases:

Fase I. Evaluación. Sesiones de 1 a 3 (tres sesiones). En la primera sesión se realizó una entrevista-filtro a los cuidadores primarios en ausencia del niño, en donde se recabó la información general del caso, tales como la descripción, caracterización y topografía de la conducta. En la segunda sesión se llevó a cabo una entrevista semiestructurada al niño en ausencia de los cuidadores primarios para indagar lo necesario respecto a su sintomatología, pensamientos y emociones asociadas. Por último, en la tercera sesión se aplicaron los dos instrumentos de evaluación elegidos: CMASR-2 y SCAS.
Fase II. Tratamiento cognitivo-conductual. Sesiones 4 a 17 (trece sesiones de tratamiento y una de cierre). La sesión 4 se dedicó a brindar psicoeducación únicamente a los cuidadores primarios sobre las causas y manifestaciones de la ansiedad que el menor presentaba. Asimismo, se ofrecieron algunas estrategias y sugerencias sobre el manejo, mismas que se llevarían a cabo en el tratamiento psicológico.

A partir de la sesión 5, los diez minutos iniciales se dedicaban a monitorear la evolución del menor durante la semana previa, y en los diez minutos finales el niño explicaba a su cuidador lo aprendido durante la sesión; la terapeuta resolvía dudas y dejaba la tarea de aplicar lo aprendido al menos en una situación cotidiana. Los cuarenta minutos 
restantes de las sesiones 5 a la 16 se dedicaron al niño, durante las que aprendía estrategias cognitivo-conductuales para disminuir su ansiedad, entre las cuales se encuentra la psicoeducación, dada en la primera sesión, en la que se hacía referencia a la película Intensa-Mente (Rivera, 2015) para ubicar las emociones y así comenzar a mostrar la ansiedad como una emoción semejante al temor, pero con características específicas. Se les explicaba el circuito de "falsa alarma" y se identificaban las sensaciones físicas corporales, así como los pensamientos asociados.

El niño se entrenaba también en técnicas de relajación, particularmente en respiración diafragmática y en relajación autógena acompañada de imaginación guiada, mediante la cual se producían sensaciones mentales de calor y tranquilidad cada vez que se sentía ansioso.

Otra estrategia fue la identificación de pensamientos positivos y negativos a través de dibujos, para posteriormente reconocer las sensaciones que cada uno de estos generaban en su cuerpo; cuando eran negativos y rumiativos, se le enseñaba la técnica de detención de pensamiento mediante la palabra "¡Alto!”, al tiempo que se daba un golpe sobre la mesa y se orientaba la conducta hacia la respiración diafragmática.

El paciente se entrenaba también para que se proporcionara instrucciones y afirmaciones positivas tales como "Yo puedo", "Voy a hacer mi mejor esfuerzo", "Estoy haciendo un gran trabajo", "Calma, todo está bien" o "Tranquilo, estás en un lugar seguro", las que decía en voz alta, acariciando sus manos y cerrando los ojos.

Para modificar las distorsiones cognitivas, se subrayaba la comprobación de los pensamientos negativos y su fundamentación en la realidad. Asimismo, se ejecutaban ejercicios, tales como encuestar a las demás personas, investigar en medios electrónicos de modo que permitieran comparar sus ideas respecto a lo que ocurre en realidad; se llevaban a cabo ejercicios contra la vergüenza, hechos con disfraces, o con el uso de analogías para explicar situaciones preocupantes, pero que se encontraban fuera de control. Por ejemplo, con ayuda del llamado "pastel de la responsabilidad", se aprendía que dicha situación puede tener diversos factores que la provocan, algunos de los cua- les dependen del niño, no así otros, por lo que se entrenaba también en estrategias de solución de problemas.

El uso del humor fue también una técnica realizada con el paciente mediante la ridiculización de los estímulos generadores de ansiedad (un asaltante, un payaso, un monstruo...), elaborando dibujos graciosos y generando a la vez respuestas incompatibles con la ansiedad.

En algunas ocasiones -dependiendo del nivel cognitivo del paciente- el debate sobre las creencias se hacía mediante el diálogo, con confrontaciones directas y empleando el humor ante las discrepancias en su pensamiento. Se hacía uso además del juego de roles, el modelamiento, las situaciones simuladas y los ensayos conductuales a fin de aplicar lo aprendido al contexto cotidiano.

En la sesión 17 se programó el cierre del tratamiento y se hizo con el paciente, de una forma más específica, un listado de los ejercicios llevados a cabo en las sesiones y las conclusiones a las que se llegaron. Se realizó también el postest mediante la aplicación de los instrumentos CMASR-2 y SCAS.

Fase III. Seguimiento. Sesiones 18 y 19 (dos sesiones). La sesión 18 se programó pasado un mes de concluido el tratamiento, y la sesión 19 dos meses después. Al inicio de cada una de estas sesiones se realizó una entrevista no estructurada tanto al cuidador primario como al menor, para monitorear el mantenimiento de los cambios, al tiempo que se resolvían sus dudas. Posteriormente, ya solo con el niño, se recordaban algunas estrategias para resolver las dificultades a las que se hubiera enfrentado durante el tiempo de seguimiento, y se terminaba con la aplicación de los seguimientos 1 y 2 .

\section{RESULTADOS}

Para comparar los resultados, se identificaron las puntuaciones naturales obtenidas por los participantes en cada uno de los instrumentos, las cuales se muestran en la Tabla 2.

En la tabla se puede observar el contraste entre las posiciones ocupadas por los casos 1, 9 y 10 en ambos instrumentos, en donde el primero de ellos fue quien obtuvo la mayor diferencia de 
puntuaciones entre el pretest y el seguimiento 2, y quien mostró la mayor mejoría. Por el contrario, los casos 9 y 10 fueron quienes obtuvieron la menor diferencia y, por tanto, la mejoría más pobre.

Tabla 2. Puntuaciones naturales obtenidas en ambos instrumentos.

\begin{tabular}{|r|r|r|r|r|c|l|l|l|l|r|c|}
\hline \multicolumn{1}{|l|}{ CMASR-2 } & \multicolumn{10}{l|}{ SCAS } \\
\hline Caso & Pre & Post & Seg1 & Seg2 & $\begin{array}{l}\text { Diferencia } \\
\text { Pre-Seg2 }\end{array}$ & Caso & Pre & Post & Seg1 & Seg2 & $\begin{array}{l}\text { Diferencia } \\
\text { Pre - Seg2 }\end{array}$ \\
\hline 1 & 35 & 24 & 23 & 13 & 22 & 1 & 101 & 55 & 44 & 49 & 52 \\
\hline 2 & 27 & 17 & 16 & 8 & 19 & 8 & 61 & 30 & 15 & 21 & 40 \\
\hline 3 & 23 & 10 & 17 & 6 & 17 & 6 & 43 & 16 & 31 & 13 & 30 \\
\hline 4 & 27 & 9 & 9 & 12 & 15 & 4 & 36 & 11 & 16 & 16 & 20 \\
\hline 5 & 18 & 13 & 1 & 8 & 10 & 7 & 22 & 22 & 3 & 4 & 18 \\
\hline 6 & 19 & 10 & 17 & 9 & 10 & 5 & 35 & 33 & 21 & 20 & 15 \\
\hline 7 & 14 & 17 & 4 & 4 & 10 & 2 & 30 & 21 & 18 & 15 & 15 \\
\hline 8 & 21 & 19 & 9 & 13 & 8 & 3 & 26 & 18 & 15 & 13 & 13 \\
\hline 9 & 11 & 6 & 13 & 7 & 4 & 9 & 18 & 12 & 17 & 10 & 8 \\
\hline 10 & 24 & 18 & 24 & 24 & 0 & 10 & 55 & 31 & 43 & 55 & 0 \\
\hline
\end{tabular}

Nomenclatura: Pre: Pretest; Post: Postest; Seg1: Seguimiento 1; Seg2: Seguimiento 2.

La columna "Diferencia" se calculó restando Pre de Seg2. Los valores se ordenaron de mayor a menor, de acuerdo con la diferencia.

En las Figuras 1 y 2 se muestran los diagramas de caja y bigote para la observación de estas puntua- ciones de forma grupal, representando la primera el CMASR-2 y la segunda el SCAS.

Figura 1. Diagrama de caja y bigote para el CMASR-2.

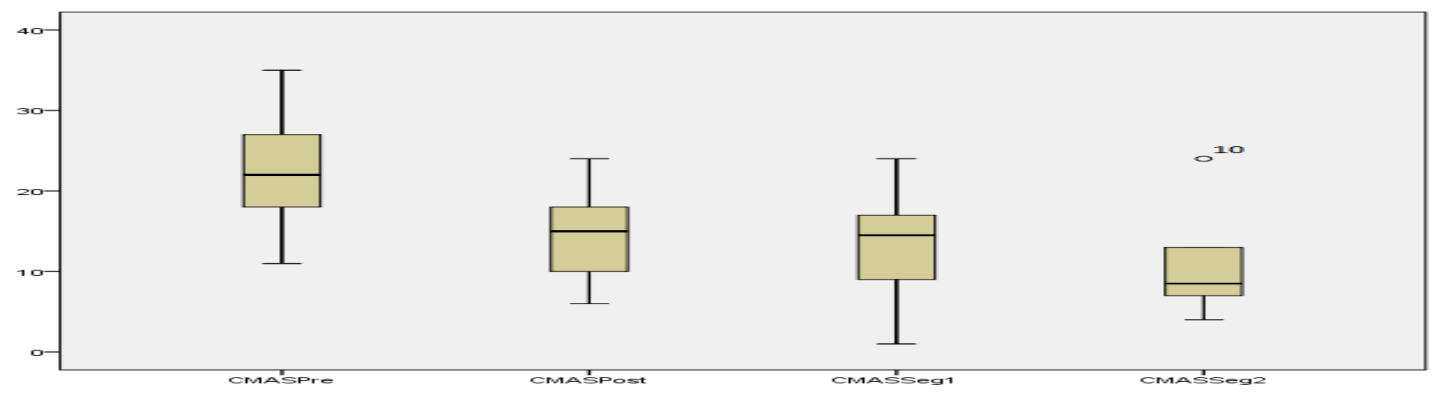

Nota: En esta figura se muestra la disminución obtenida a través de las puntuaciones naturales del CMASR-2. El caso 10 se cataloga como un caso atípico por alejarse más de 1.5 rangos intercuartílicos de la caja.

Figura 2. Diagrama de caja y bigote para el SCAS.

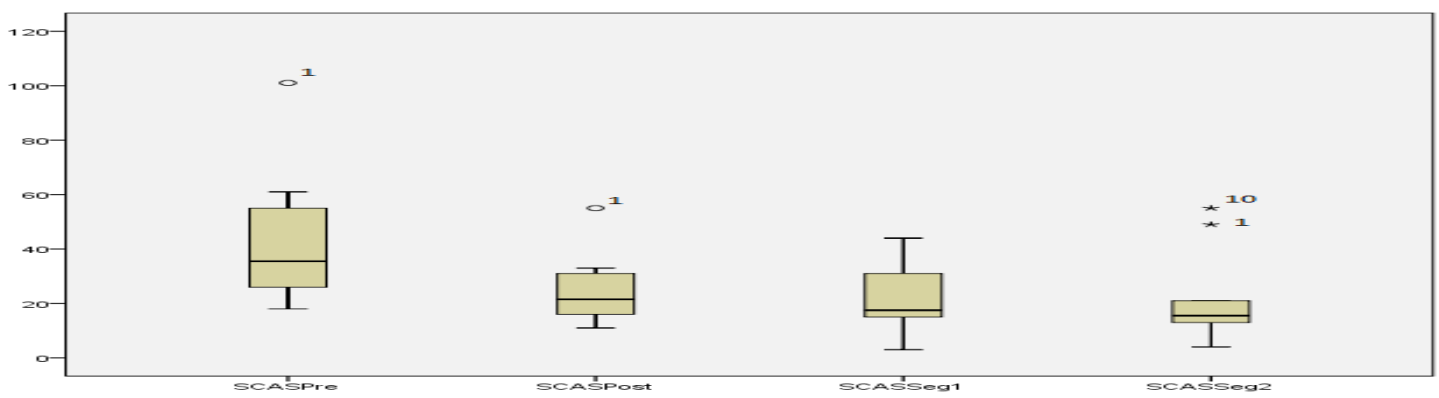

Nota: En esta figura se muestra la forma en cómo se distribuyen las puntuaciones naturales obtenidas por el instrumento SCAS. En el pretest, postest y el seguimiento 1, el caso 1 se considera atípico por alejarse más de 1.5 rangos intercuartílicos de la caja. En el seguimiento 2 , los casos 10 y 1 se consideran extremos por alejarse más de 3 rangos de esta. 
La Figura 1 representa la reducción gradual de las puntuaciones máximas y mínimas obtenidas en el CMASR-2; en el seguimiento 1 se observa todavía una reducción mayor de la puntuación mínima (el bigote inferior es aún más largo). En el último seguimiento, la puntuación máxima se orienta hacia la media del resto de las puntuaciones (no asoma a la caja), y la puntuación mínima se acerca a ella, denotando menor dispersión. Sin embargo, se observa un caso atípico; en relación a la Tabla 2, la puntuación del caso 10 sale de esta distribución y se mantiene aún en términos elevados.

En la Figura 2 también se puede observar una reducción de las distribuciones de la gravedad de la ansiedad, aunque más sensible a los valores atípicos. Por ejemplo, la caja y los bigotes del postest son considerablemente menores a los observados en el pretest, y aunque en el seguimiento 1 las puntuaciones máximas y mínimas se extienden mostrando una mayor dispersión, también son menores al pretest. En el seguimiento 2 es todavía más visible esta reducción; al igual que el CMASR-2, la puntuación máxima (bigote superior) se integra a la media del resto de las puntuaciones. En el pretest, postest y el seguimiento 1, el caso 1 sale de las distribuciones por tener puntuaciones mayores que el resto, y fue también el que tuvo la mayor mejoría. En el último seguimiento, los casos 1 y 10 se consideran extremos, pues este último se aleja aún más del percentil 75, en comparación con el CMASR-2.

La disminución gradual de las puntuaciones en el CMASR-2 y en el SCAS se traduce en la reducción de las creencias que los participantes tuvieron en relación con la exposición de sí mismos ante el juicio o la evaluación de los otros -por ejemplo, hacer el ridículo ante un examen, cometer errores en una exposición o sufrir las burlas que puede generar el hacer nuevos amigos-; de igual manera, se redujeron los síntomas de nerviosismo, la dificultad para dormir o la sudoración de manos. De manera específica, en el SCAS también se refleja la reducción de la sensación de pérdida de control, la creencia de perder la razón o de morir, así como la inseguridad percibida respecto a que algo negativo ocurra mientras se está solo, ser ridiculizado por los otros frente a una situación social, o ser asaltado o dañado por alguien en el contexto externo, entre otros.

El reporte verbal de estos cambios fue dado tanto por el paciente como por su cuidador en momentos distintos. En siete casos, el cambio ocurrió durante el TCC, y no mientras se experimentaba el efecto de la farmacoterapia, y en tres casos de forma simultánea a la farmacoterapia y al curso de la TCC, por lo que en estos últimos no fue posible identificar qué factor propició el cambio.

Para evaluar los resultados antes y dos meses después de finalizado el tratamiento, se calcularon los estadísticos descriptivos entre la medida del pretest y el seguimiento 2 en ambos instrumentos. Los datos indican una reducción de la media de aproximadamente $50 \%$, pues en el caso del CMASR-2 la media de puntuaciones se redujo de 42.7 a 21.6, la puntuación mínima disminuyó de 18 puntos a 4 , y la puntuación máxima de 101 a 55. En el caso del SCAS, la media disminuyó de 21.9 a 10.20 , la puntuación mínima se redujo de 11 a 4 puntos y la máxima de 35 a 24 .

Para evaluar si este cambio fue significativo se llevó a cabo la prueba de rangos con signo de Wilcoxon. La Tabla 3 señala los resultados arrojados en cada uno de los instrumentos.

Tabla 3. Prueba de rangos con signo de Wilcoxon. Frecuencias para el CMASR-2 y el SCAS.

\begin{tabular}{|l|c|c|c|c|}
\hline Instrumento & $\begin{array}{c}\text { Diferencias } \\
\text { negativas }^{\mathbf{a}}\end{array}$ & $\begin{array}{c}\text { Diferencias } \\
\text { positivas }^{\mathbf{b}}\end{array}$ & Empates $^{\mathbf{c}}$ & Total \\
\hline CMASR-2 & 9 & 0 & 1 & 10 \\
\hline SCAS & 9 & 0 & 1 & 10 \\
\hline
\end{tabular}

Nota: El superíndice significa: a: Seguimiento $2<$ Pretest; b: Seguimiento $2>$

Pretest, y c: Seguimiento $2=$ Pretest.

En la tabla se puede apreciar que nueve participantes tuvieron diferencias negativas o de disminución de puntuaciones en la comparación seguimiento 2 - pretest, y solo un participante obtuvo un empate; es decir, su puntuación fue igual en ambas aplicaciones de los instrumentos. Los estadísticos de prueba arrojados para el CMASR-2 corresponden a lo siguiente: $\mathrm{w}=-2.67, p=0.007, p<0.05$; 
y similares en el SCAS: $\mathrm{w}=-2.66, p=0.008, p<$ 0.05 , lo que significa que hay una diferencia significativa en los puntajes de ansiedad que el grupo de participantes obtuvo en ambos instrumentos.

\section{DISCUSIÓN}

El objetivo del presente trabajo fue evaluar el efecto del TCC en un grupo de diez niños de 8 a 11 años de edad, diagnosticados con trastornos de ansiedad, lo cual se logró según los resultados obtenidos.

La comparación de las puntuaciones obtenidas durante el pretest, el postest y los seguimientos permitió identificar una reducción de la sintomatología de los participantes antes y después del tratamiento; esto es, se modificaron ciertos síntomas físicos asociados a los episodios de ansiedad, tales como sudoración en las manos o dolores de estómago, creencias irracionales al someterse al juicio o la evaluación de los otros, o de que algo negativo podría ocurrirles si salían a la calle o si se quedaban solos, etc. De acuerdo con lo señalado por Ellis y Grieger (1981) y Beck (2000), al debatir las creencias erróneas o distorsionadas y al sustituirlas por otras más objetivas y realistas, las emociones perturbadoras se reducen y se convierten en adaptativas, lo que constituye el eje medular del procedimiento llevado a cabo en este estudio. De hecho, se ha reportado que al entrenar a los niños en técnicas de respiración diafragmática, solución de problemas y psicoeducación respecto de lo que les sucede, así como la participación de los cuidadores primarios en el reconocimiento de los síntomas de ansiedad fisiológica, social y generalizada, favorece que la sintomatología se reduzca a niveles adaptativos (Aydin, 2014; Creswell et al., 2014; James, James, Cowdrey, Soler y Choke, 2013; Olivares et al., 2003; Seligman y Ollendick, 2011).

Estos resultados son consistentes con el cálculo obtenido de la comparación entre el seguimiento 2 y el pretest y los datos arrojados por la prueba de rangos con signo de Wilcoxon para evaluar su significancia estadística, pues se observó que hubo una reducción de alrededor de $50 \%$ en la media de las puntuaciones naturales de ambos instrumentos; las puntuaciones mínimas y máximas obtenidas fueron menores en el seguimiento 2, y nueve participantes lograron una reducción de sus síntomas antes y después del tratamiento, lo que implica una reducción significativa de acuerdo con los estadísticos de prueba arrojados y al mantenimiento, e incluso mejora, de los resultados.

Una variable de difícil control a lo largo del procedimiento fue que todos los pacientes se encontraban bajo tratamiento farmacológico desde su primer contacto con el hospital según su historia clínica, por lo que el TCC llevado a cabo siempre estuvo acompañado de dicha condición. Sin embargo, en siete de los participantes los cambios en la sintomatología fueron reportados de manera verbal por el niño y por sus cuidadores durante el tratamiento, y no así cuando el efecto del medicamento estaba vigente. Uno de los participantes reportó únicamente en mantenimiento de los síntomas y no el empeoramiento detectado en la Fase I de evaluación, en cuyas mediciones reflejó las más altas puntuaciones. En tres de los participantes el reporte verbal de mejoría ocurrió de forma simultánea a los efectos de la farmacoterapia y la TCC, por lo que no fue posible saber si uno u otro factor, o su combinación, generaron el cambio observado.

Los niños reportaron cambios en relación a sus creencias, la forma en cómo percibían los sucesos que antes provocaban ansiedad y la manera en cómo podían manejarlos, lo que es consistente con lo que diversos autores han hallado respecto a la TCC como elemento importante para modificar este tipo de síntomas, pese a haber un tratamiento farmacológico asociado (Aydin, 2014; Creswell et al., 2014; Gil y Hernández, 2009; Gutiérrez y Gutiérrez, 2015; James et al., 2013; López, 2012; Olivares et al., 2003; Peña y Ramos, 2009; Seligman y Ollendick, 2011).

Si bien se ha demostrado que si se emplea la combinación de TCC con inhibidores selectivos de la recapturación de serotonina (ISRS) se reducen aún más los síntomas de ansiedad (Creswell et al., 2014; Ipser et al., 2009; Sawyer y Nunez, 2014), también se ha visto que únicamente la TCC ha resultado efectiva para este tipo de padecimientos en dicha población (Aydin, 2014; Creswell et al., 2014; Gil y Hernández, 2009; Gutiérrez y Gutiérrez 2015; James et al., 2013; López, 2012; Olivares et al., 2003; Peña y Ramos, 2009; Seligman y Ollendick, 2011), lo que siembra la duda de si los 
mismos resultados se hubieran obtenido sin la farmacoterapia. De acuerdo con ello, es posible que tales resultados sugieran que los síntomas ocurrieron en niveles todavía mayores a los reportados cuando los niños se incluyeron en este estudio por el efecto supresor del fármaco, por lo que la farmacoterapia únicamente habría logrado una mejoría parcial. Sin embargo, no existen mediciones en estos casos que permitan confirmarlo.

La importancia de evaluar la efectividad de la TCC por separado radica en la preocupación por los efectos secundarios de la ingesta de medicamentos, el tiempo necesario de su consumo y el costo que implican a las familias e instituciones (Cárdenas et al., 2010; Creswell et al., 2014; Martínez et al., s.f.; Reid et al., 2015), por lo que se sugiere que en estudios posteriores se hagan ajustes al diseño metodológico mediante grupos experimentales con los tratamientos por separado, y grupos control que permitan evaluar si se obtendrían resultados similares en ausencia de la farmacoterapia, y así tener una mayor claridad del alcance de cada uno de estos tratamientos; sin embargo, ello tendría que acordarse con la institución en la cual se lleven a cabo el procedimiento y con las familias implicadas.

En cuanto a los instrumentos empleados en este estudio, se rescata la importancia de contar con dos escalas que midan el mismo constructo y con las cuales se puede verificar la confiabilidad en los datos obtenidos, lo que proporciona un mayor soporte metodológico a este estudio al obtenerse resultados similares en ambos instrumentos. Se destaca también la ventaja del SCAS al contar con una escala tipo Likert de cuatro opciones de respuesta, lo que facilita que el menor gradúe me- jor sus respuestas con base en lo que le ocurre y no solo elija entre respuestas dicotómicas, como es el caso del CMASR-2, lo cual al investigador también le resulta más conveniente para valorar la evolución progresiva de los síntomas.

Finalmente, los resultados derivados de este diseño metodológico permitieron identificar que la reducción de la sintomatología se mantuvo a través del tiempo (hasta dos meses después del término del tratamiento). Será conveniente que en estudios posteriores se plantee el seguimiento durante un periodo mayor para así conocer el tiempo real en que se mantienen los efectos.

En conclusión, a pesar de que los participantes siempre estuvieron bajo el tratamiento farmacológico indicado por su médico paidopsiquiatra tratante, es posible identificar que la TCC fue efectiva dado que en la mayoría los cambios sucedieron a partir de su curso y no cuando el efecto del medicamento estaba vigente. Se pudo modificar las creencias irracionales respecto a los eventos disparadores de ansiedad y se indujo el aprendizaje de estrategias que hicieron posible su manejo óptimo. Tales resultados son consistentes con las reducciones estadísticamente significativas de las puntuaciones obtenidas en los instrumentos utilizados.

El uso de ambos instrumentos puede ser una tarea especialmente compleja en niños que muestran comorbilidad con TDAH, pues implica tiempos prolongados de atención y concentración sostenida. Se sugiere que en futuras investigaciones con este tipo de población se reconsidere la pertinencia de usar ambos instrumentos, o bien se empleen en diferente número de las sesiones.

\section{REFERENCIAS}

American Psychiatric Association (2013). Diagnostic and Statistical Manual of Mental Disorders (5th ed). Washington, D.C.: American Psychiatric Association.

Aydin, A. (2014). Parental involvement in cognitive-behavioral therapy for children with anxiety disorders. Turkish Journal of Psychiatry, 25(3), 181-189.

Beck, J. (2000). Introducción. En J. Beck /Ed.): Terapia cognitiva (trad. A. Ruiz) (pp. 17-30). Barcelona: Gedisa.

Beesdo, K., Knappe, S. y Pine, D., (2009). Anxiety and anxiety disorders in children and adolescents: developmental issues and implication for DSM-V. Psychiatric Clinics of North America, 32(3), 483-524.

Benjamin, C., Podell, J., Mychailyszyn, M., Puleo, C., Tiwari, S. y Kendall, P. (2010). Terapia cognitiva comportamental para la ansiedad en los niños: Componentes clave. En M. Gomar, J. Mandil y E. Bunge (Comps.): Manual de terapia cognitiva comportamental con niños y adolescentes (pp. 207-239). Buenos Aires: Polemos. 
Cárdenas, E., Feria, M., Palacios, L. y de la Peña, F. (2010). Guía clínica para los trastornos de ansiedad en niños y adolescentes. México: Instituto Nacional de Psiquiatría Ramón de la Fuente y Secretaría de Salud.

Creswell, C. y Waite, P. (2016). Recent developments in the treatment of anxiety disorders in children and adolescents. Evidence Based Mental Health, 19(3), 65-69.

Creswell, C., Waite, P. y Cooper, P. (2014). Assesment and management of anxiety disorders in children and adolescents. Archives of Disease in Childhood, 99, 674-678. doi: 10.1136/archdischild-2013-303768.

Ellis, A. y Grieger, R. (1981). Manual de terapia racional emotiva (v.1). Bilbao (España): Descleé de Brouwer.

Gil B., F. y Hernández G., L. (2009). TCC para niños mexicanos con fobia social. Anuario de Psicología, 40(1), 89-104.

Gutiérrez G., A.V. y Gutiérrez L., M. (2015). Efectos de una intervención cognitivo-conductual para trastornos de ansiedad en niños escolares: Estudio de caso. Memorias del Congreso Mexicano de Psicología (pp. 1022-1025), Cancún, Q.R. (México), 7-9 de octubre.

Hernández G., L., Bermúdez O., G., Spence, S., González, M., Martínez G., J., Aguilar, J. y Gallegos, J. (2010). Versión en español de la escala de ansiedad para niños de Spence (SCAS). Revista Latinoamericana de Psicología, 42(1), 13-24.

Hospital Psiquiátrico Infantil “Dr. Juan N. Navarro”. Morbilidad de Consulta Externa, Enero-Diciembre 2014. México: Autor.

Ipser, J., Stein, D., Hawkridge, S. y Hoppe, L. (2009). Pharmacotherapy for anxiety disorders in children and adolescents. Cochrane Database of Systematic Reviews, 3(CD005170), 555-628. doi: 10.1002/14651858.CD005170.pub2.

James, A., James, G., Cowdrey, F., Soler, A. y Choke, A. (2013). Cognitive behavioural therapy for anxiety disorders in children and adolescents (Review). Cochrane Database of Systematic Reviews, 6(CD004690), 1-104. doi: 10.1002/14651858. CD004690.pub3.

Keeton, C.P., Kolos, A.C. y Walkup, J.T. (2009). Pediatric generalized anxiety disorder: epidemiology, diagnosis and management. Pcediatric Drugs, 11(3), 171-183. doi: 10.2165/00148581-200911030-00003.

López F., A. (2012). TCC en el trastorno de ansiedad generalizada en pacientes del Psiquiátrico Infantil Juan N. Navarro. (Tesina inédita de Licenciatura). México: UNAM.

Martínez O., A., Fernández A., C., Navarro M., I. y Martínez M., M. (s.f.). Guía clínica. Diagnóstico y manejo de los trastornos de ansiedad. Guías clínicas del Hospital Psiquiátrico Infantil “Dr. Juan N. Navarro”. México: Secretaría de Salud.

Medina-Mora, M., Borges, G., Lara, M.C., Benjet, C., Blanco, J.J., Fleiz B., C., Villatoro V., J., Rojas G., E., Zambrano R., J., Casanova R., L. y Aguilar G., S. (2003). Prevalencia de los trastornos mentales y uso de servicios: Resultados de la Encuesta Nacional de Epidemiología Psiquiátrica en México, Salud Mental, 26(4), 1-16.

Olivares, J., Rosa, A., Caballo, V., García L., L., Orgilés, M. y López G., C. (2003). El tratamiento de la fobia social en niños y adolescentes: Una revisión meta-analítica. Psicología Conductual, 11(3), 599-622.

Peña, A. y Ramos, L. (2009). Ansiedad infantil: Presentación de un caso y su tratamiento. Tesis inédita de Licenciatura en Psicología. México: UNAM.

Polanczyk, G., Salum, G., Sugaya, L., Caye, A. y Rohde, L. (2015). Annual research review: A meta-analysis of the worldwide prevalence of mental disorders in children and adolescents. Journal of Child Psychology and Psychiatry, 56(3), 345-365.

Reid, A., McNamara, J., Murphy, T., Guzick, A., Storch, E., Geffken, G. y Bussing, R. (2015). Side-effects of ssiRs disrupt multimodal treatment for pediatric OCD in a randomized controlled trial. Journal of Psychiatric Research, 71, 140-147. doi: 10.1016/j.jpsychires.2015.10.006.

Reynolds, R.C. y Richmond, O.B. (2012). CMASR-2: Escala de Ansiedad Manifiesta en Niños Revisada (Trad. G. Vélez). México: El Manual Moderno.

Rivera, J. (Productor) y Docter, P. y Del Carmen, R. (Dirs.) (2015). Intensa-Mente [Película]. Los Ángeles, CA: Pixar Animation Studios y Walt Disney Pictures.

Sawyer, M. y Nunez, D. (2014). Cognitive-behavioral therapy for anxious children: from evidence to practice. Worldviews on Evidence Based Nursing, 11(1), 65-71.

Secretaría de Salud, Organización Panamericana de la Salud y Organización Mundial de la Salud. (2011). Informe sobre el sistema de salud mental en México. México: Autores. Recuperado de http://www.inprf.gob.mx/opencms/export/sites/INPRFM/ psicosociales/archivos/iesm_oms.pdf.

Seligman, L. y Ollendick, T. (2011). Cognitive behavioral therapy for anxiety disorders in youth. Child and Adolescent Psychiatric Clinics of North America, 20(2), 217-238. doi: 10.1016/j.chc.2011.01.003.

Spence, S.H. (1997). Structure of anxiety symptoms among children: A confirmatory factor-analytic study. Journal of Abnormal Psychology, 106, 280-297.

World Health Organization (2004). Prevention of mental disorders. Effective interventions and policy options. Geneve: WHO. Recuperado de http://www.who.int/mental_health/evidence/en/prevention_of_mental_disorders_sr.pdf. 